\title{
Investigation on Rock Segmentation and 3D Surface Evaluation Based on Binocular Vision
}

\author{
Hongwei Gao ${ }^{1 *}$, Ben $\mathrm{Niu}^{2,3}$, Bin $\mathrm{Li}^{1}$, Yang $\mathrm{Yu}^{1}$ \\ ${ }^{1}$ School of Information Science \& Engineering, Shenyang Ligong University, Shenyang, 110159, China \\ ${ }^{2}$ Hefei Institute of Intelligent Machines, Chinese Academy of Sciences Hefei, 230031, China \\ ${ }^{3}$ College of Management, ShenzhenUniversity Shenzhen, 518060, China \\ *Corresponding author's Email: ghw1978@sohu.com
}

\begin{abstract}
Planetary exploration is the embodiment of a nation's synthesis science and technology power. The first step is to land a rover to explore the unknown world. The science task always begins from rock and soil. Rock sample and return is one of the important tasks of planetary exploration, which should be accomplished by the rover's hardware and software. A series of algorithms related to main science exploring object-rock are investigated in this paper, such as a single rock's segmentation, shadow elimination, stereo matching and 3D reconstruction. C-means clustering and 3D surface evaluation realized by surrounding binocular vision are described. On the basis of the above algorithms, a virtual mechanism is developed by $\mathrm{VC}++$ and OpenGL. The evaluated information for localization is transformed to the arm and leads the arm to locate the relative plane on the rock and shows the grinding process. The experiments and simulation results based on real image show the validity of these algorithms.
\end{abstract}

Keywords: Rock Segmentation, 3D Reconstruction, C-means clustering, 3D Surface Evaluation

\section{Introduction}

The U.S.A decided to return to the moon in 2005 , whose main task is to achieve a soft landing of the moon's surface probe. The JPL, as one of the units that planned and developed the project, puts forward the moon's surface obstacle detection technology based on passive image. This technology which uses passive visual image through the relevant recognition algorithm is aimed at detecting the obstacles independently such as craters, faults, slopes and rocks, etc, which affect the safe landing of the probe. At the same time, Chinese lunar exploration engineering has formally commenced with project and implementation and the second phase of moon exploration will launch a lunar probe landing on the moon. One of the important means of implementing this goal is lunar rover. At present, a number of domestic universities and re- search institutes have carried out some preliminary research in lunar rover and have achieved some results. They are 502 Aerospace Science and Technology Corporation, Beijing University of Aeronautics and Astronautics, Tsinghua University and Harbin Institute of Technology, etc [1]. To achieve lunar rover's roaming is the basis of other follow-up work, and the topographic information provided by the stereo vision system is the key step of lunar rover to achieving secure roaming. The stereo vision system can also provide three-dimensional information of the rock to be examined for vehicle manipulator. Currently how rover adopted human-computer interaction mode to determine the rocks attracts most scientists' interests. Experts determines the rocks to be examined in the twodimensional images, then the rocks are reconstructed and its reconstruction surface is evaluated automatically by the algorithms, after that, the results are fed 
back to the experts who will decide the manipulator's final location and grind surface. The human-computer interaction mode is more secure, accurate, and reliable and thus becomes the basis of finally achieving automatic working way. Recently, the rock detection algorithm based on the passive image mainly includes: the method based on the edge information [1], the method based on texture analysis [2], the method based on shadow detection [3] and the method based on solid geometry, etc [4]. This paper mainly investigates the segmentation algorithm of a single rock in the image, matching algorithm and three-dimensional reconstruction algorithm, 3D surface evaluation algorithm, and the simulation of mechanism arm located on the relative plane on the rock gotten by surface evaluation algorithm. All the algorithms are based on the passive image gotten from the earth ground circumstances. The simulation results show that the proposed algorithms are correct and effective.

\section{Rock segmentation}

In computer vision systems, there exist many shadows in most scenes. The shadow is produced when the direct light is blocked wholly or partly by the object. Although people can identify the shadow easily from objects, it is still a difficulty to identify shadows by the computer. Detection and elimination of shadows are necessary to be done before the further algorithm processing because the shadow can influence effectiveness and correctness of subsequent algorithm in image segmentation, target tracking and identification and $3 \mathrm{D}$ reconstruction.

\subsection{Shadow elimination}

To the rock-the location object of stereo vision in the paper, the 3D reconstruction of its shadow and itself within a scene may be processed if we don't consider the influence of shadow. The lack of abundant grayscale information can't reflect the true deep information, and a large number of error matches will be produced during matching. Ultimately the $3 \mathrm{D}$ reconstruction result of rock surface will be influenced, so that subsequent evaluation results of rock surface will be affected indirectly. All above, it will affect mechanical arm to locate and grind the rock.

Detecting shadow accurately is a difficult job; the points of target and shadow have a similar visual effect in many cases, especially in dealing with the grayscale image only. Therefore, researchers have begun to consider joining the color information to improve the shadow detection rate. However, the cameras used on lunar vehicle are all black and white cameras which can only capture gray-scale images for the consideration of limitation of communication traffic. For this reason, many shadow detection algorithms based on color will not be used. This paper will only study shadow detection and separation algorithm based on gray-scale image.

The irradiating angle of the rock varies with the different position of two cameras, so the shadow size of rock which is exposed in the sun will be different in left image and right image. Moreover, the shading will also cause the different shadow size and the gray scale also varies with different rocks. As a result it will not work if we adopt fixed threshold segmentation or binaryzation by thinking of shading case comprehensively under lighting condition.

The constraint condition of the algorithm is as follows:

1. The lunar vehicle usually works at noon when the illumination angle of sunlight is a measurable range, so gray value and distribution range of the shadow are small. It is easy to combine the shadow into the same area if dividing the area according to pixel value.

2. The area ratio of shadow region and normal rock without shadow can be roughly determined through ground experiment, i.e. the number ratios can be determined by their respective share of pixels.

The detailed steps of shadow elimination algorithm are as follows:

1. Obtain the VGH from left and right image (the local image after the rock area being determined manually).

2. The distribution number of shadow pixels is determined probably by combining with prior knowledge (which is measured by ground experiment), and its gray value is used as the segmentation threshold of rock shadow.

3. The shadow pixels are separated from the selected region and the cross correlation matching is only used to process non-shadow pixels.

In step (2), the gray value of shadow region in general is smaller and its number is less than the gray value of rock surface, so the distribution number of shadow pixels is determined by prior knowledge (which is measured by ground experiment). In step (3), after the 
shadow pixels separation, a judgment condition is added to gray value of pixels during the process of matching. The cross correlation matching is processed pixel-bypixel in the outer loop, the inner judgment compute cross correlation of the pixels whose gray value is not within gray value range of shadow pixels, which can reduce the amount of computation. The experimental

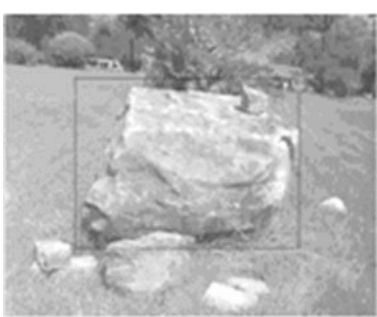

(a) Original image

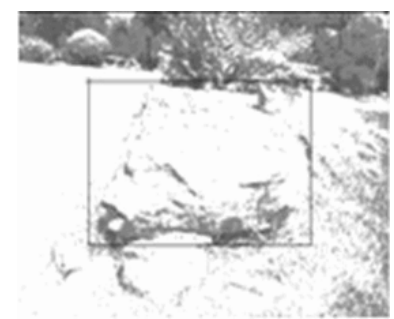

(b) Shadow mark after binarizaiton
Figure 1. Shadow eliminate

results are shown as figure 1; the shadow pixels under the rock have been processed through the binarization and have been marked. In spite of the pixels which meet the condition obtain the pixels of rock surface (The situation such as color and shading of the rock itself will produce the pixels whose gray value are low and similar to the pixels of shadow pixels), but they are few, there are little effects on the subsequent rock surface reconstruction by processing them as shadow pixels.

\subsection{Segmentation algorithm of the rock}

The grinding surface can be fitted automatically according to the three-dimensional data of reconstructed rock. Then, the burnisher on the front of manipulator can be guided to locate on the grinding plane by the spatial normal vectors of fitting plane as well as the three-dimensional coordinates of anchor point. After verification in a virtual environment, the manipulator's joint information is passed to the real manipulator, and guides the manipulator to grind and sample on the rocks. As the lunar vehicle uses the humancomputer interaction mode operation generally, one can determine the general location of the rock manually (drag a rectangle on the image), and separate the area occupied by the shadow of the rock by using fixed threshold segmentation or binarization approach. Then an accurate segmentation and matching will be done for the same rock.

\subsubsection{Segmentation based on cluster}

The separation method based on the cluster can be used if the boundary of rock and background is not obvious [5]. First, one can estimate an approximation plane equation of the ground, calculate the vertical distance from all space points to the plane, and then process the k-means clustering according to the elements of the distance. There are two types of data. One is the ground data with smaller distance, and the other is the rock surface data as shown in figure2. Subsequently, one can fit equation of the ground plane, separate the ground data and rock surface data once more. Finally, the grinding plane can be fitted automatically according to $3 D$ data of rock reconstruction. The $3 D$ coordinates of the points selected manually (just click once in picture, the corresponding coordinate is evaluated by $u_{2}^{T} F u_{1}=0$ in the other picture, then $3 D$ reconstruction of the point can be done) can be used as the initial cluster center. The rough separa-

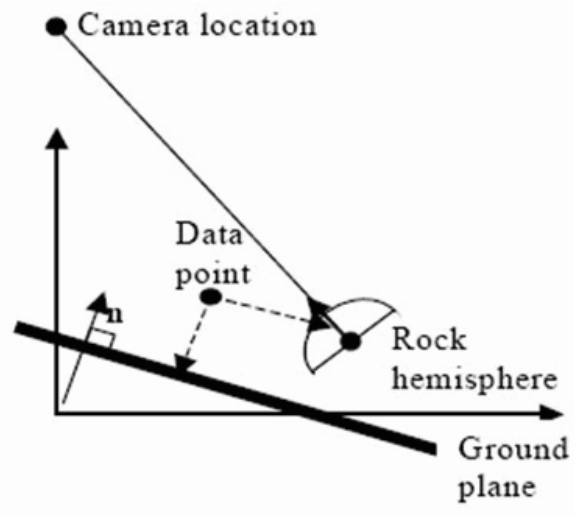

Figure 2. Model of rock and ground

tion algorithm of a single rock is as follows:

1. Estimate an approximation plane equation of the ground according to the three-point method and the three points are at the bottom corner area in the two images (They are usually on the ground).

2. Calculate the vertical distance from all space points to the plane, then process the k-means clustering according to the elements of the distance (apparently, there are only two classes, ground and rock). One class distance is smaller (ground) and the other one is bigger (the rock surface data).

3. Then fit equation of the ground plane again after separating the ground data and rock surface data:

$$
\left\{\begin{array}{l}
A X_{1}+B Y_{1}+C Z_{1}+D=0 \\
\cdots \ldots \ldots \ldots \\
A X_{n}+B Y_{n}+C Z_{n}+D=0
\end{array}\right.
$$


The value of $A, B, C$ and $D$ is calculated by means of least square method with $3 D$ point coordinate and the point cloud number is represented by $n$.

4. Classify the original data again according to the distance between points and plane on the basis of the new ground equation, and recalculate the ground plane equation until the distance between the vectors of two adjacent plane equation meet:

$$
d=\sqrt{\left(A-A^{c}\right)^{2}+\left(B-B^{c}\right)^{2}+\left(C-C^{c}\right)^{2}+\left(D-D^{c}\right)^{2}}<\varepsilon
$$

Where, $\varepsilon$ is a very small threshold value.

5. Get the vector of approximative plane.

\subsubsection{Segmentation based on feature constraint}

The segmentation of rock data will be processed after the shadow being processed within the selected region. The separation methods based on edge will be adopted if the boundaries of rock and background are clear. First, one can detect the edge of the rock by using edge detection of the background, select the areas of the rock manually, and then extract the edge data within the region of areas. Matching and 3D reconstruction of non-edge points can be processed in interior of edge under the restriction of edge data. Matching can be achieved fast by means of dynamic programming.

The steps of matching algorithm in local image are based on the feature constraint:

1. Select the areas manually in which the rock located, and determine its size as well position of each vertex.

2. Detect the edge points by using Canny algorithm, and just retain the edge points within the area.

3. Find the edge points of each scanning line, and match the points based on gray correlation in the same scanning line of the two images. Considering the constraint of order guarantee, one can sort edge points respectively which are located in the scanning line of the left and right image by compositor of $\mathrm{u}$ direction, and then match them.

4. The edge points between two sides are divided into several areas by the matching pairs. The matching of non-feature points will be done under the constraint of known edge parallax.

5. Exclude the wrong matches.

\section{3D Reconstruction of rock}

\subsection{Calibration of binocular vision}

Calibration will be done in the high-precision calibration platform by using Tsai two-step classic method which takes the radial distortion into account. There are two kinds of parameters and both of them need to be calibrated. The internal parameter represents the geometry and optical properties of the image sensor. The external parameter determines the position between the camera and the world coordinate system. The first step of Tsai two-step method is to calibrate $R$ which is $3 \times 3$ rotation matrix, scaled factor $s_{x}$, the first two elements $t_{x}$ and $t_{y}$ of translation matrix. The second step is calibrating focal length $f, t_{z}$ and radial distortion $k_{1}$. First, calculate the value of $f$ and $t_{z}$ in the case of $k_{1}=0$, and then calculate the optimal value iterative according to RAC model. Adopt corner detection algorithm accurate to sub-pixel level. This will ensure the average measuring error of stereo vision system within $1 \mathrm{~mm}$ in three directions $(X, Y, Z)$ after calibrating.

\subsection{Stereo Matching}

Considering the matching speed and accuracy, dense matching algorithm based on graph cut is adopted in this paper [6]. Gray information is as one of the items in global optimizing object function, so the disparity map is little affected by gray difference and more accuracy than the disparity map gotten by gray crosscorrelation. The global optimizing object function is defined as follows:

$$
E(f)=E_{\text {data }}(f)+E_{\text {occ }}(f)+E_{\text {smooth }}(f)
$$

Where, $E_{\text {data }}$ is data item which represents consistency degree for corresponding matching pixels. $E_{o c c}$ is the penalty item for ensuring every pixel to find matching as possible as it can. $E_{\text {smooth }}$ is the smooth item for restricting the consistent disparity between neighbor pixels. This problem belongs to combination optimizing problem and can be solved by max flow-min cut theory. According to this theory, the min cut of a network can be achieved by means of calculating max flux. The construction of the network is made of fountain point, converge point, disparity side and smooth side as shown in figure 3 . Every side has different capacity (cost). Thus, the matching problem is equivalent to finding the network's min cut, while the bend surface for all sides running through by min cut is called min cut bend surface. All the capacity (cost) of the sides on the surface is minimum, the bend 


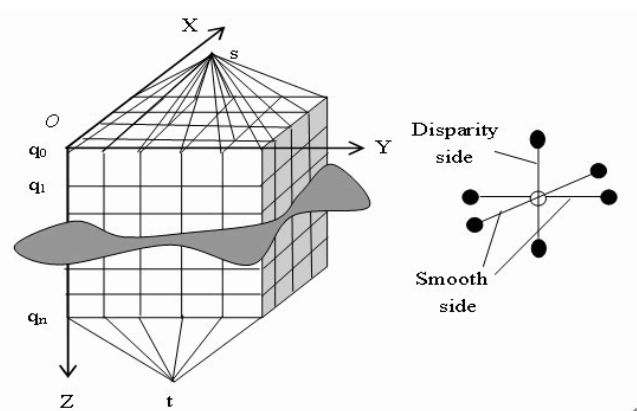

Figure 3. Definition of Network flux

surface can be considered as the disparity $d$ changing according to the image coordinate $(x, y)$ within the overlap area between two images.

\subsection{D Reconstruction}

3D measurement of space points is often adopted triangulation principle. In this paper, we reconstruct the space points with the camera parameters based on distortion model.

$$
\left\{\begin{array}{l}
s_{x}^{-1}\left(1+k_{1} r^{2}\right) X_{d i}=f \frac{r_{11} x_{w i}+r_{12} y_{w i}+r_{13} z_{w i}+t_{x}}{r_{31} 1 w_{w i}+r_{32} y_{w i} i+r_{33} z_{w i}+t_{z}} \ldots \\
\left(1+k_{1} r^{2}\right) Y_{d i}=f \frac{r_{21} x_{w i}+r_{22} y_{w i}+r_{23} z_{w i}+t_{y}}{r_{31} x_{w i}+r_{32} y_{w i}+r_{33} z_{w i}+t_{z}} \cdots
\end{array}\right.
$$

Where, $s_{x}$ is the scale factor, $k_{1}$ is the radial distortion coefficient, $R$ is the $3 \times 3$ rotation matrix, $t_{x}, t_{y}$ and $t_{z}$ are the elements in the translation vector $T$. The elements of $R$ denoted by $r_{i j}(i=13, j=13)$. $\left(X_{d}, Y_{d}\right)$ is the actual image coordinate, and its underneath corner sign $i$ is the number of calibration points.

The specific process is as follows: Set $A=s_{x 1}^{-1}(1+$ $\left.k_{1}\left(d u^{2}\left(u_{1}-u_{01}\right)^{2}+d v^{2}\left(v_{1}-v_{01}\right)^{2}\right)\right) d u\left(u_{1}-u_{01}\right)$ According to the RAC model given above and expressing the left part of the equation 3(a) by A, we will obtain:

$$
\begin{array}{r}
\left(A r 1_{31}-f_{1} r 1_{11}\right) x_{w i}+\left(A r 1_{32}-f_{1} r 1_{12}\right) y_{w i}+ \\
+\left(A r 1_{33}-f_{1} r 1_{13}\right) z_{w i}=f_{1} t_{x 1}-A t_{z 1}
\end{array}
$$

Set

$$
B=\left(1+k_{1}\left(d u^{2}\left(u_{1}-u_{01}\right)^{2}+d v^{2}\left(v_{1}-v_{01}\right)^{2}\right)\right) d v\left(v_{1}-v_{01}\right)
$$

Expressing the left part of the equation 3(b) by B, we can get:

$$
\begin{aligned}
\left(B r 1_{31}-\right. & \left.f_{1} r 1_{11}\right) x_{w i}+\left(B r 1_{32}-f_{1} r 1_{12}\right) y_{w i}+ \\
+ & \left(B r 1_{33}-f_{1} r 1_{13}\right) z_{w i}=f_{1} t_{x 1}-B t_{z 1}
\end{aligned}
$$

In the same way, we can get:

$$
\begin{aligned}
& C=S_{x 2}^{-1}\left(1+k_{2}\left(d u^{2}\left(u_{2}-u_{02}\right)^{2}+\right.\right. \\
& \left.\left.\quad+d v^{2}\left(v_{2}-v_{02}\right)^{2}\right)\right) d v\left(v_{2}-v_{02}\right) \\
& \left(C r 2_{31}-f_{2} r 2_{11}\right) x_{w i}+\left(C r 2_{32}-f_{2} r 2_{12}\right) y_{w i}+ \\
& \quad+\left(C r 2_{33}-f_{2} r 2_{13}\right) z_{w i}=f_{2} t_{x 2}-C t_{z 2}
\end{aligned}
$$

Set $D=\left(1+k_{2}\left(d u^{2}\left(u_{2}-u_{02}\right)^{2}+d v^{2}\left(v_{2}-v_{02}\right)^{2}\right)\right) d v\left(v_{2}-\right.$ $\left.v_{02}\right)$, we can get:

$$
\begin{array}{r}
\left(D r 2_{31}-f_{2} r 2_{11}\right) x_{w i}+\left(D r 2_{32}-f_{2} r 2_{12}\right) y_{w i}+ \\
+\left(D r 2_{33}-f_{2} r 2_{13}\right) z_{w i}=f_{2} t_{y 2}-D t_{z 2}
\end{array}
$$

Take equations (4) to (7) into consideration, we will obtain:

$$
\begin{array}{r}
{\left[\begin{array}{ccc}
A r 1_{31}-f_{1} r 1_{11} & A r 1_{32}-f_{1} r 1_{12} & A r 1_{33}-f_{1} r 1_{13} \\
B r 1_{31}-f_{1} r 1_{21} & B r 1_{32}-f_{1} r 1_{22} & B r 1_{33}-f_{1} r 1_{23} \\
C r 2_{31}-f_{2} r 2_{11} & C r 2_{32}-f_{2} r 2_{12} & C r 2_{33}-f_{2} r 2_{13} \\
D r 2_{31}-f_{2} r 2_{21} & D r 2_{32}-f_{2} r 2_{22} & D r 2_{33}-f_{2} r 2_{23}
\end{array}\right]} \\
{\left[\begin{array}{l}
x_{w i} \\
y_{w i} \\
z_{w i}
\end{array}\right]=\left[\begin{array}{l}
f_{1} t_{x 1}-A t_{z 1} \\
f_{1} t_{y 1}-B t_{z 1} \\
f_{2} t_{x 2}-C t_{z 2} \\
f_{2} t_{y 2}-D t_{z 2}
\end{array}\right]}
\end{array}
$$

corresponding $\left(x_{w i}, y w i, z_{w i}\right)$ can be solved by least square method.

\subsection{Triangulate partition}

A group of 3D point cloud data obtained by 3D reconstruction can not reflect the details of the entire surface. Therefore, it is necessary to triangulate these 3D points; the surface of object can be composed of many small triangles. The minimum interior angle of each triangular element in the grid is an important standard of measuring the grid quality: If the minimum interior angle is the biggest, the grid quality is better, while Delaunay triangulations being exactly the optimal triangulations which meet that the minimum interior angle is the biggest [7]. Solving Delaunay triangulation algorithm is mainly circumscribed circles criterion, local optimization criterion, etc. Circumscribed circles optimization criterion is a kind of common criteria of solving the Delaunay triangulations, and it is also the grid optimization criterion used in this paper.

In actual application, the triangulation of $2 \mathrm{D}$ plane (the result is a set of triangles) and the triangulation of 3D space (the result is a set of tetrahedron) are used most commonly. The algorithm in the paper is designed for the triangulations of $2 \mathrm{D}$ plane. The triangulations are processed separately based on the matching points between left image and right image, then the triangulation lines among points are mapped to the 3D space and formed 3D triangular mesh. It can also approximate to the real shape of object even by adopting seldom triangle if the matching is accurate. After triangulations, all of the triangular planar chips make up 3D model of the object in the space. Then the only work is to take texture of object out of the image and to map it to 3D model, thus the real model of object is obtained. 


\section{Rock surface evaluation}

\subsection{C-means Clustering}

The grinding object is appointed by operator, so the rock with flat surface usually is selected because it can be distinguished by human eye, and the number of surface which approximates flat surface can also be judged by naked eye. That makes it possible to the application of clustering algorithm and to the realization of automatic evaluation algorithm for the rock surface.

C-means algorithm is a kind of dynamic clustering method. This algorithm only uses one clustering center as the representative of one class, but one point cannot fully reflect the distribution structure of the class so that a lot of useful information is lost. Therefore, $\mathrm{C}$ class of $\mathrm{C}$-means algorithm should be adjusted [8], then nucleoid replaces the class center and advanced C-means algorithm is adopted to improve its capability of classification.

The steps of C-means Clustering algorithm are as follows:

1. The $\mathrm{C}$ class will be produced from initial division for the given set of eigenvector to be classified. Assume that the set of eigenvector to be classified has been standardized $x_{1}, x_{2}, \ldots, x_{N}$, where $N$ is the total of the eigenvector to be classified, dividing $C$ class is to be desired. Let eigenvector $x_{t}=\left(x_{1 t}, x_{2 t}, x_{3 t}\right)^{T}$ (The clustering in the paper is based on $3 D$ data, so each eigenvector have three components). Define $\operatorname{sum}(i)=$ $\sum_{m=1}^{3} x_{m t}$, and set $M A=\max _{i}[\operatorname{sum}(i)], M I=\min _{i}[\operatorname{sum}(i)]$, then $a_{i}$ can be computed as follows:

$$
a_{i}=\frac{(c-1)[\operatorname{sum}(i)-M I]}{M A-M I}+1, i=1,2, \ldots, N
$$

Obviously, $1 \leq a_{i} \leq c, x_{i}$ is divided to $w_{j}$ if $a_{i}$ near the integer. To process all samples like above can realize initial classifications, thereby, we obtain the initial clustering center $z_{1}^{(k)}, z_{2}^{k}, \ldots$, $z_{c}^{(k)}$, where $k=0, k$ represents the number of iterations. Where $z_{j}^{(k)}=\frac{1}{n_{j}^{(k)}} \sum_{x_{i} \in w_{j}^{(k)}} x_{i}, n_{j}$ is the number of eigenvector in each clustering $w_{j}$.

2. Compute mean vector and $u_{j}$ and covariance matrix $\sum_{j}$ of each clustering $w_{j}$ according to the below formulas.

$$
\begin{array}{r}
u_{j}=\frac{1}{u_{j}} \sum_{x_{i} \in w_{j}} x_{i} \\
\sum_{j}=\frac{1}{n_{j}} \sum_{x_{i} \in w_{j}}\left(x_{i} x_{i}^{T}-u_{j} u_{j}^{T}\right)
\end{array}
$$

3. Divide each sample $x_{i}$ to a certain class according to the principle of minimum distance. $\mathrm{Nu}-$ cleoid can replace class center, one can define a nucleoid function $K_{j}=K\left(x, V_{j}\right)$ to represent distribution of the class $w_{j}$. Where $V_{j}$ is a parameter set about class $w_{j}, x$ is a sample. In order to describe the degree of closeness between $x$ and $w_{j}$,one can define the distance:

$$
\begin{aligned}
& d\left(x, w_{j}\right)=\ln \left\|\sum_{j}\right\|+ \\
& \quad+\left(x-u_{j}\right)^{T} \cdot \sum_{j}^{-1}\left(x-u_{j}\right)-2 \ln \frac{n_{j}}{N}(11)
\end{aligned}
$$

Where $\left|\Sigma_{j}\right|$ is the determinantal value of $\sum_{j}$, and $\left\|\sum_{j}\right\|$ is the absolute value of $\left|\sum_{j}\right|, \sum_{j}^{-1}$ is the inverse matrix of $\sum_{j}$.

If $d\left(x, w_{k}\right)=\min _{j}\left\lfloor d\left(x, w_{j}\right)\right\rfloor$, then $x \in w_{k}$.

4. Compute the new clustering center:

$$
z_{j}^{k+1}=\frac{1}{n_{j}^{(k+1)}} \sum_{x_{i} \in w_{j}^{(k+1)}} x_{i}
$$

If there are no samples to change its class, that is $z_{j}^{(k+1)}=z_{j}^{k}(j=1,2, \ldots, c)$, then the algorithm stops, otherwise jumps to step (2).

\subsection{The step of algorithm about evaluation of rock surface based on $\mathrm{C}$-means clustering}

1. Judge the number of similar flat surfaces on the rock surface according to observing the rock image, and take this as the input of clustering analysis algorithm (There are two or three classes in general).

2. Solve the centroid and normal vector of all triangular plates which make up the rock surface.

3. Every normal vector is clustered, and the angle among vectors is selected as principle of optimization, and any two normal vectors are given as the initial center vector of two classes. If the angle is less than 5 degree, there exist two initial classes.

4. Recompute normal vector of centroid in every class(the mean value of angel between all normal vector of every class and every axis), and use this as the center normal vector of next clustering. Clustering goes on until the angle of two clustering center vector doesn't change.

5. Output center normal vector of clustering and corresponding 3D coordinate of centroid. 
After rock segmentation, the grinding surface is fitted automatically according to $3 \mathrm{D}$ data of reconstructed rock (several planes that may be located on them are given), and the space normal vector of fit plane is used to guide manipulator burnisher to locate on the grinding surface. After space plane (triangle) is determined, the normal vector of plane possibly being operated is passed to manipulator and guides the manipulator to approach the almost flat surface to be operated along the direction of vector. Through 3D measurement, it is converted into $3 D$ coordinate $(X, Y, Z)$ within world coordinate system according to the determined three points $A\left(X_{A}, Y_{A}, Z_{A}\right), B\left(X_{B}, Y_{B}, Z_{B}\right)$, $C\left(X_{C}, Y_{C}, Z_{C}\right)$. Take two of these vector randomly such as $A B$ and $A C$, so the cross product between $A B$ and $A C$ is the plane normal vector that is determined by three points. So barycentric coordinates of triangle $A B C$ can be obtained by mean value of corresponding component.

\subsection{Realization of the virtual mechanism arm}

The graphic simulation is always applied in 3D reconstruction and virtual reality, otherwise, it can also be used to design and verify the robot system, such as robot programming, control and tele-operation. OpenGL is an open 3D graphic library for developing highquality graphic program. It can also be combined with Visual $\mathrm{C}++$ program tool. The virtual mechanism arm used in this paper is drawn by OpenGL. There are

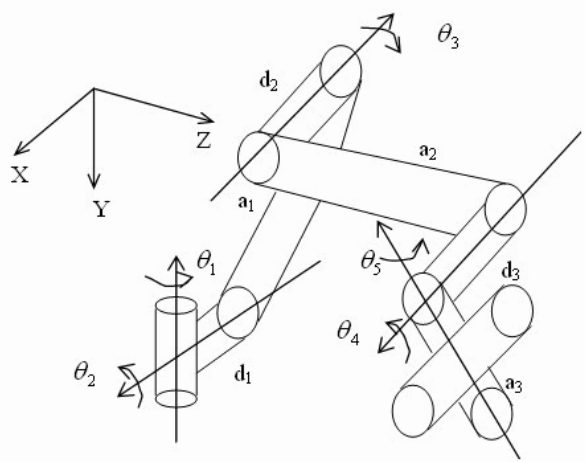

Figure 4. Definition of mechanism arm joint angle

four degrees in the arm, which are shoulder base joint, great arm rotate joint, little arm rotate joint, wrist rotate joint and hand rotate joint. They are represented by $\left(\theta_{1}, \theta_{2}, \theta_{3}, \theta_{4}, \theta_{5}\right)$. The initial position of the arm is $(00145350)$, the unit is degree. Every joint angle's range is (090090014535180-4545). The definition of the angle's direction is shown in figure 4 . The direction of $\mathrm{Z}$ axis is vertical with the computer screen and points to its inside. Then, the mechanism arm's kine- matical solution and invert solution can be deduced.

\section{Experiment results and analysis}

The binocular vision system is Bumblebee 2 made by PointGrey company whose resolution is 1024768 . The length of the baseline is $150 \mathrm{~mm}$, the calibration job is finished on the accurate calibration platform, the software is developed by own with $\mathrm{VC}++$.

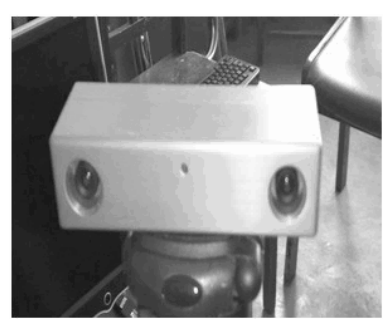

(a) Experimental equipment

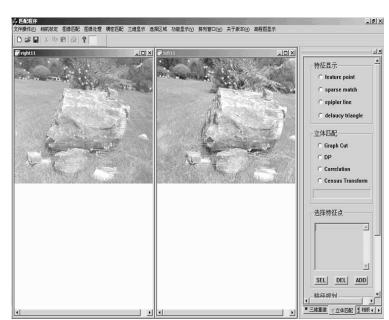

(b) stereo vision software
Figure 5. Equipment and Software platform

Table 1. Results in the rock evaluation process

\begin{tabular}{|c|c|c|c|}
\hline Scence & $\begin{array}{c}\text { Disparity } \\
\text { rangeafter } \\
\text { rectification }\end{array}$ & $\begin{array}{c}\text { Dense matching } \\
\text { Disparity mean } \\
\text { value filter } \\
\text { before-behind }\end{array}$ & $\begin{array}{c}\text { Grind } \\
\text { plane } \\
\text { number }\end{array}$ \\
\hline Rock & -2120 & $23465-21382$ & 2 \\
\hline
\end{tabular}

Figure 5 shows the experimental equipment and selfdeveloped stereo vision software. Figure 6 shows the simulation results of local feature matching, $3 D$ reconstruction, $3 D$ surface evaluation for rock area and the robot arm localization process on the rock plane. Figure 6(a) shows the rock's area selected through humancomputer interaction, the algorithms talked above are executed in this area. Figure 6(b) is the results after epipolar line rectification, the matching points now are in the same line which can reduce the calculation quantity and improve the matching accuracy, and the disparity range is -2120 pixels (seen form table 1). Figure 6(c) is the result of the image edge detection through Canny detector, figure 6(d) is the disparity map of the rock in the selected area under the edge constraint. Effectiveness of the algorithms can be seen from these figures.

Figure 6(e) is the results of dense matching. There are 23465 points in the rectangle area (seen form table 1), after disparity mean value filter, there are 21382 points left which can be used to triangulation partition. Figure 6(f) shows the triangulation partition result in 2D image, the green lines are the borders of numerous triangles. When the $3 \mathrm{D}$ reconstruction is finished, 


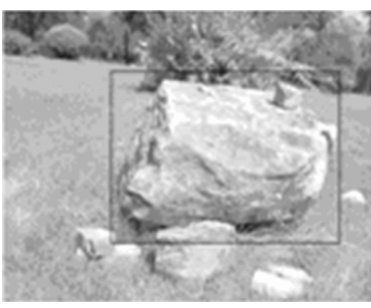

(a) Original image

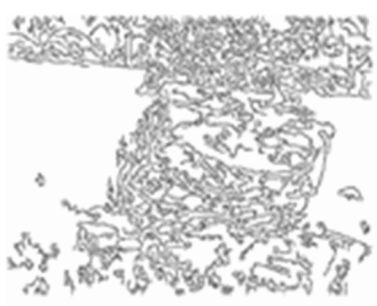

(c) Edge detection

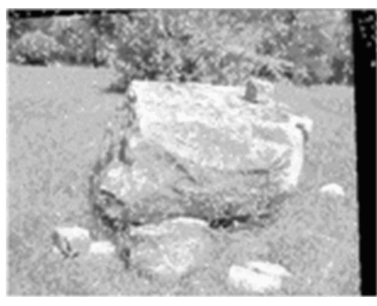

(e) Dense matching

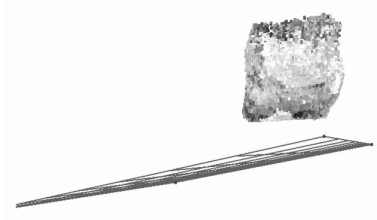

(g) 3D point cloud

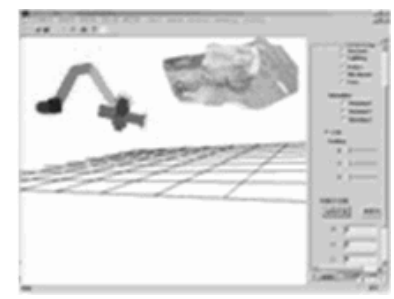

(i) Robot arm deploy

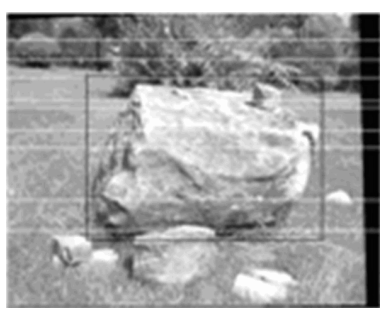

(b) Epipolar line rectification

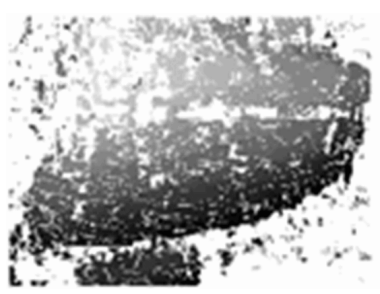

(d) Dispairty map of rock

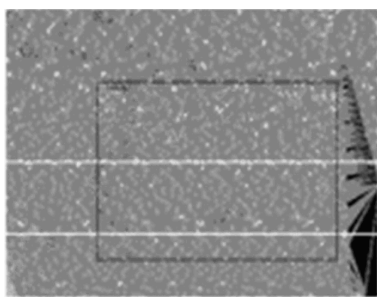

(f) Triangulation partition

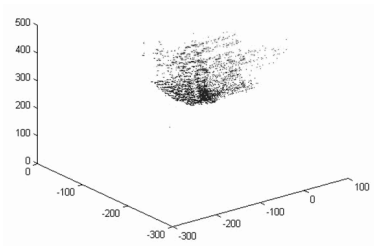

(h) Evaluation result by clustering

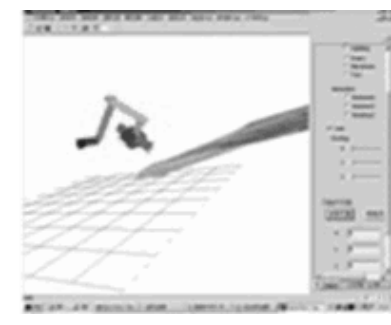

(j) Robot arm localization
Figure 6. Experiment results

the triangle in the 2D image can be projected into 3D space and form the 3D triangle, the 3D surface of the rock can be restored after texture stick.

Figure $6(\mathrm{~g})$ is the $3 \mathrm{D}$ point cloud of the rock, for the reason of the dense of thousands of points; the 3D shape of the rock can be seen. Figure 6(h) is the evaluation result based on the cluster of the angle berween normal vector of neighbor triangular plate. There are two kinds of points presented by red and blue respec- tively. Therefore, there exist two possible localization planes for robot arm to grind (seen form table 1).

Figure 6(i) and figure 6(j) show the robot arm localization simulation results. According to the evaluation results from figure 6(f), we get two planes used to grind which is represented by red and blue points. The figure 6(i) and figure 6(j) is the simulation process based on plane calculated from the red points. When the robot kinematics model is determined, then the simulation can be started with exhibition of the arm deploy. The simulation results prove the validity of the series algorithms discussed in this paper.

\section{Conclusion}

Under the background of planetary exploration, this paper discusses the investigation on the scientific research object-rock, such as 3D reconstruction and 3D surface evaluation technology of a single rock in the image. Locating the virtual mechanism arm to the relative plane on the rock evaluated by the above technologies is feasible and proved by the experiment and simulation results, and relational algorithms are also effective. These technologies can provide technical support for scientific investigation of the rover, especially for the lunar rover of our country. The future work will focus on segmentation and evaluation of several rocks automatically and designing algorithms suitable for lunar circumstance.

\section{Acknowledgments}

This work is supported by China Liaoning Provice Educational Office fund (No.20080611). National Natural Science Foundation (No.71001072).

\section{References}

[1] R. Castano, M. Judd, T. Estlin, etal, "Onborad Autonomous Rover Science”, In: Proc. IEEE Aerospace Conference, Big Sky, Montana: Pasadena, CA, pp.113, 2007.

[2] Y. H. Song, "Automated Rock Segmentation for Mars Exploration Rover Imagery", the 39th Lunar and Planetary Science Conference, League City, Texas, pp.2043-2051, 2008.

[3] V. C. Gulick, R. L. Morris, M. A. Ruzon, "Autonomous Image Analysis During the 1999 Marsrokhod Rover Field Test”, Geophysical Research, vol.106, pp.7745-7764, 2001.

[4] R. T. David, S. Trey, W. David, "Data Mining During Rover Traverse: From Images to Geologic Signatures", the 8th International Symposium on Artificial Intelligence, Robotics and Automation in Space, 
Haus der Bayerischen Wirtschaft Munchen, Germany, pp.324-335, 2005.

[5] L. Pedersen, "Science target assessment for Mars rover instrument deployment", IEEE/RSJ International Conference on Intelligent Robots and System, vol.1, pp. 817- 822, 2002.

[6] V. Kolmogorov, R. Zabih, "Computing visual correspondence with occlusions using graph cuts", the Eighth International Conference on Computer Vision, pp.508-515, 2001

[7] J. Y. Liu, P. D. Zhou, "Triangulation is no t the M inimum Weight Triangulation", Journal of Computer Aided Design \& Computer Graphics, vol.13, no.12, pp. 1150-1152, 2001 (in Chinese).

[8] M. Alata, M. Molhim, and A. Ramini, "Optimizing of Fuzzy C-Means Clustering Algorithm Using GA”, World Academy of Science, Engineering and Technology, vol.39, pp.224-229, 2008. 